\title{
Relación entre estilos educativos familiares y el valor social subjetivo de educación: reflexiones a partir de un estudio
}

\author{
Relationship between family educational styles and social value \\ of education: reflections of a study
}

\author{
María Jesús Perales Montolío \\ Mercedes Bisquert Martínez \\ Margarita Bakieva \\ Universitat de València
}

Recibido: $24 / 10 / 2018$

Aceptado: 10/12/2018

\begin{abstract}
The goal of this study is to analyze the relationship between the social value that the families give to education of their sons and daughters, and the family educational styles. For this, we apply 4103 questionnaires of evaluation of Family Educational Styles (EEF) of Bisquert (2017). We used 5 context variables of the same questionnaire because of their conceptual relationship with the evaluation dimensions of Subjective Social Value of Education (VSESubjective) of Jornet, Perales and Sánchez Delgado (2011). The association was estimated by crosstab procedure, and Pearson's chi-square coefficient. The results reveal the significant association in two of the four dimensions of VSESubjective, but with a low intensity (between 0.103 and 0.155 ).
\end{abstract}

KEY WORDS: social value of education; family education; assessment indicators; educational evaluation.

\section{RESUMEN}

El objetivo del presente estudio es analizar la relación entre el valor social que le otorgan las familias de alumnos de primaria y secundaria de la Comunidad Valenciana y Castilla y León a la educación de sus hijos e hijas y los estilos educativos que adoptan con ellos. Para ello, se han recogido 4103 cuestionarios para conocer datos respecto a cuál es el valor que atribuyen a la educación, y cómo lo trasmiten a sus hijos e hijas. Se aplicó el instrumento de evaluación de Estilos Educativos Familiares (EEF) de Bisquert (2017). Por otra parte, utilizamos 5 variables de contexto del mismo cuestionario por su relación conceptual con las dimensiones de evaluación de VSE-Subjetivo Jornet, Perales y Sánchez Delgado, 2011). Se estudió la asociación mediante el procedimiento de tablas de contingencia y el cálculo de coeficiente chi-cuadrado de Pearson. Los resultados muestran la asociación significativa en dos de las cuatro dimensiones que estructuran el concepto de VSE-Subjetivo, aunque con una intensidad baja (entre 0.103 y 0.155 ).

PALABRAS CLAVE: valor social de educación; estilos educativos familiares; educación familiar; evaluación de la educación.

\section{Introducción}

Las evaluaciones de sistemas educativos muestran diferencias entre países, entre regiones, entre grupos sociales, respecto a los resultados escolares (OCDE, 2018). Las familias tienen un papel fundamental en el proceso escolar y educativo de sus hijos, entre otras cosas, por el valor que atribuye a la educación como clave de desarrollo personal y social de éstos.

Para centrar la mirada en educación, indistintamente sea ésta entendida como un proceso de desarrollo y crecimiento personal a lo largo de la vida, o sea considerada sólo la fase de su desarrollo dentro del ámbito formal, es primordial contemplar en primer lugar la familia, calificada como el primer espacio educativo, no sólo porque precede en el tiempo a cualquier otra instancia educativa, sino también en cuanto a su potencial en la formación de los individuos (Cánovas y Sahuquillo, 2010);

Dirección de correspondencia:

María Jesús Perales Montolío, Universitat de València, Depto. de Métodos de Investigación y Diagnóstico en Educación. E-mail: perales@uv.es._ORCID: https://orcid.org/0000-0003-20332750 
además de ser conscientes de las necesidades actuales que presentan. En su estudio sobre el análisis sobre las intervenciones socioeducativas con las familias desde diferentes contextos, Cánovas, Sahuquillo, Císcar, y Martínez (2014) justifican la necesidad de potenciar actuaciones de apoyo y protección a la familia y la infancia, dada la diversidad de situaciones y problemáticas en que se ven inmersas.

Por todo ello y por todos los cambios emergentes a nivel social y relación entre iguales, será de gran interés observar qué valor otorga la familia a la educación como elemento de desarrollo personal y social.

La apuesta que niños, niñas y adolescentes hagan por la educación, el esfuerzo que le dediquen, el compromiso que asuman, va a depender del valor que ellos y ellas, y sus familias, le concedan como elemento útil para su propia felicidad, y para lo que ellos y ellas consideren éxito vital. Esto es a lo que se ha dado en llamar Valor Social de la Educación (VSE).

El VSE, de acuerdo con la definición de Jornet et al. (2011) se define como:

La utilidad que tiene la educación dentro de una sociedad para el desarrollo y la promoción de las personas en los ámbitos social y laboral, así como a las ventajas que aporte como elemento de prevención de la exclusión social y como garantía para el desarrollo y la mejora de su bienestar a lo largo de la vida (p. 52).

Históricamente, estudiar estuvo sólo al alcance de algunos. La segunda mitad del siglo XX trajo la democratización de la educación, primero a unos territorios y luego a otros. Todavía la total cobertura es un reto por conseguir en algunas partes del mundo, y especialmente para algunos colectivos, como las niñas, las adolescentes, y en muchos casos las zonas rurales. Así se recoge en los Objetivos para el Desarrollo Sostenible (OCDE, 2015). La particularidad del momento actual, la tragedia, es que algunos colectivos, en algunos territorios, ya no ven claramente la educación como clave para la movilidad social y para el propio desarrollo personal y social. En unos casos, porque los índices de desempleo son intolerablemente altos; en otros, además, porque la imagen social que se da del éxito es tan arbitraria y vinculada a valores superfluos, que resulta difícil seguir manteniendo ante los y las adolescentes el discurso del valor del esfuerzo y de la educación. En este contexto, es fundamental el papel de la familia en la lectura crítica de estos mensajes; es clave su papel apostando por el valor social de la educación.

El concepto de VSE se define desde dos perspectivas, la objetiva y la subjetiva. El VSEObjetivo se define como

la utilidad real que tiene la educación, dentro de una sociedad, para el desarrollo y la promoción de las personas en los ámbitos social y laboral, así como las ventajas que tenga para ellas como elemento de prevención de la exclusión social, y como garantía para el desarrollo y la mejora de su bienestar a lo largo de la vida (Jornet et al., 2011, p. $53)$.

La apuesta objetiva de una sociedad por la educación se mide, entre otras cosas, en cómo la financia, cómo la recoge en sus presupuestos, tanto por el porcentaje de gasto que les dedica, como la cantidad final que aporta (Jornet et al., 2011). La evidencia muestra que la apuesta de un país por la selección, la formación, la motivación de su profesorado, tanto a nivel inicial como durante su desarrollo profesional, va a ser clave para la calidad de la educación, tal y como señala el Informe MacKinsey (Mourshed, Chijioke y Barber, 2012). Pero las situaciones de crisis pueden poner en cuestión esta apuesta social por la educación. Así, en el mundo occidental en general, y en España de una forma especialmente intensa, la crisis económica iniciada en 2008 se ha traducido en severos recortes en educación, que han incrementado sustancialmente la situación de desigualdad (OCDE, 2018). La apuesta social por la educación de los gobiernos se ha reducido, y todavía no se ha recuperado (Schraad-Tischler y Schiller, 2016; Lázaro Lorente, 2017); y la sociedad, como globalidad, no es totalmente consciente de ello. 
Paralelamente, el VSE-Objetivo de las familias, la apuesta objetiva de las familias por la educación, se podría medir por su inversión en la educación de sus hijos e hijas: inversión en términos económicos (apoyos suplementarios a la educación, actividades extraescolares y complementarias...) y, sobre todo, inversión en tiempo y dedicación, tanto al seguimiento escolar de sus hijos e hijas, como a la participación en los centros educativos a través de las Asociaciones de Madres y Padres de Alumnos/as.

La segunda dimensión del VSE es la subjetiva. Se define el VSE-Subjetivo como la percepción que los actores principales del proceso de enseñanza-aprendizaje (alumnado, familias y profesorado) tienen acerca de la importancia de la educación para la promoción social, laboral y del bienestar personal y colectivo, a lo largo de la vida (Jornet et al., 2011, p. 67).

La familia va a ser una influencia fundamental, junto con el profesorado, los iguales, y la sociedad en general, en el valor social que los y las estudiantes atribuyan a la educación. Los mensajes recibidos en casa van a modular y contextualizar otros mensajes recibidos en el contexto social o a través de los mass-media. Y esta influencia la va a ejercer a través de los estilos educativos, la transmisión de valores, el modelado ejercido respecto al compromiso con la educación y el valor del esfuerzo, la dinámica interna familiar, o incluso la educación respecto al locus de control (externo o interno) "vinculado a la formación de las expectativas y aspiraciones de logro, la explicitación de metas en el desarrollo profesional y las consecuencias sociales que ello puede conllevar para los hijos" (Jornet et al., 2011, p. 70).

En concreto, las dimensiones e indicadores que se proponen para la evaluación del VSESubjetivo de los diferentes agentes implicados, entre ellos la familia, se recogen en el Cuadro 1.

Cuadro 1.

Fuentes de recogida de información

DIMENSIÓN $\quad$ INDICADOR

Dimensión 1. Expectativas y metas educativas Nivel de aspiraciones educativas.

¿Cuáles son las aspiraciones y metas que Expresión de las metas generales de se plantean el alumnado y las familias en logro educativo que tiene el alumnado y $\quad \mathrm{X} \quad \mathrm{X}$ la educación? sus familias.

Niveles a los que se aspira llegar.

Dimensión 2. Justicia Socialy Educación. Percepción personal del alumnado ¿Qué consecuencias y/o beneficios respecto a las consecuencias de su perciben alumnado y familias que tiene esfuerzo ante las demandas educativas. la Percepción de su propia experiencia educación para el bienestar personal y como educando. social?

Percepción de las familias y del alumnado acerca del modo de acceso al éxito social y al bienestar.

Dimensión 3. Valor diferencial de la Importancia de la educación para la educación. vida: Personal, Laboral, y Social.

¿Qué valor intrínseco para la vida dan a la educación el alumnado, las familias y el profesorado?

Dimensión 4. Obstáculos y facilitadores. Percepción de los elementos o factores ¿Qué elementos les pueden ayudar o que pueden favorecer o limitar el logro dificultar en el proceso educativo? de los objetivos educativos planteados tanto por el alumnado como por sus familias.

Fuente: Dimensiones e indicadores propuestos para el VSE-Subjetivo, en J. Jornet et al. (2011). 
Uno de los elementos que participa en la formación del valor social otorgado a la educación por parte de los alumnos es el modelo de actuación o patrón con el que los padres ejercen su labor familiar; estos modelos de influencia de las familias en hijos e hijas se denominan Estilos Educativos Familiares (EEF). Los estilos educativos se vinculan con algunos indicadores que se han mostrado como clave en el concepto de formación del VSE-Subjetivo (para más información ver Bisquert, 2017).

El estudio presentado en este artículo se enmarca en el Proyecto EVALEF18, "Validación de un instrumento de evaluación de estilos educativos familiares y establecimiento de lineamientos para el diseño de programas de intervención con familias" y define EEF como "conjunto de actitudes, sentimientos y patrones de conducta que los padres y madres asumen frente al niño/a y que repercuten en el funcionamiento, tanto psicológico como social, de los hijos" (Perales, Bisquert y Sahuquillo, 2015, p. 450).

Una vez consensuado el constructo, se procede a revisar los instrumentos que analizan los EEF. Como conclusión a un largo proceso de análisis, se observa que es necesario diseñar un instrumento para aplicar a los padres que tenga en cuenta cuestiones de deseabilidad social, y a la variable derivada del contexto social y cultural.

El objetivo del presente estudio es analizar el VSE-Subjetivo de las familias, en función de los EEF que adoptan en la educación de sus hijos e hijas. Para ello, se parte del estudio realizado en el marco de la validación del instrumento, y de la información recogida respecto a variables contextuales y familiares.

El análisis de los datos recogidos de más de 4000 padres y madres, pero en algunos casos también abuelos, hermanos mayores u otras personas significativas del entorno familiar, en los contextos de Castilla y León y Comunidad Valenciana permite aportar reflexiones interesantes respecto a cuál es el valor que atribuyen a la educación, y cómo lo trasmiten a sus hijos e hijas.

\section{Método}

La metodología de nuestro estudio corresponde al estudio correlacional ex post facto. Se trata estudiar la relación entre la prevalencia de estilos educativos familiares en los padres de alumnos en función con las variables relacionadas con las dimensiones de evaluación del valor social de educación de Jornet et al. (2011).

\subsection{Muestra de estudio}

Nos basamos en una muestra de un total de 4103 de padres (un 42,1\% del total), madres $(53,1 \%)$, abuelos y abuelas $(1,3 \%)$ y otros familiares o tutores legales de alumnos matriculados en los centros escolares mayoritariamente de Primaria (un $96,3 \%$ de la muestra) y el resto de Secundaria $(3,7 \%)$. Las provincias en las que se sitúan los centros son de Alicante (5,6\% de la muestra), Palencia $(82,4 \%)$ y Valencia $(11,9 \%)$. La muestra ha sido seleccionada por criterio de disponibilidad y la mayoría de ella (un 70\% del total) ha sido recogida en los centros de titularidad pública, un $26 \%$ en los centros privados concertados, y el resto $(3,8 \%)$ en los centros privados sin concierto. La participación ha sido voluntaria y con el previo consentimiento por parte de los respondientes.

\subsection{Instrumento}

Se aplicó el instrumento de evaluación de Estilos Educativos Familiares (EEF) de Bisquert (2017). Este instrumento se compone de tres escalas: Historias Incompletas (EEF-Historias), Comportamientos habituales (EEF-Comp) y Hábitos Familiares (EEF-Hab). Para nuestro estudio utilizamos la escala EEF-Historias para diferenciar perfiles de estilos educativos familiares que adoptan los padres en su ámbito familiar. Por otra parte, utilizamos 5 variables de contexto (B03, B07, B09, B10, B11) y una variable utilizada en la escala EEF-Hábitos (B31), por su relación

18 Financiado en el Plan Nacional de I+D+I, Referencia EDU2011-29467. IP: M. J. Perales Montolío. 
conceptual con las dimensiones de evaluación de VSE-Subjetivo. Debido a que el instrumento de evaluación de EEF de Bisquert (2017) en su totalidad no es el objeto de este estudio, ya que pretende alcanzar unos objetivos mucho más globales que nos permite el espacio de este artículo, para conocer su contenido invitamos a los lectores a consultar el trabajo a través de la referencia al final de este artículo. Las definiciones de las variables del contexto utilizadas en nuestro estudio se mencionan en el apartado de resultados, agrupadas en las dimensiones del VSE-Subjetivo señaladas en el Cuadro 1.

\subsection{Procedimiento}

Se realizó este estudio dentro del marco del proyecto más amplio, descrito anteriormente en el apartado de Introducción. A partir de la escala de Historias Incompletas (EEF-Historias) se han agrupado los sujetos en función del grupo de su posición, determinada ésta por el conglomerado (clúster de $\mathrm{k}$ medias) de pertenencia, utilizando las puntuaciones de la escala para diferentes estilos educativos. Para el mayor detalle se ofrece el proceso de cálculo y validación de la escala en Bisquert (2017). Se utilizó como solución final la opción de 4 conglomerados o grupos.

Para analizar la relación entre el perfil educativo familiar y las dimensiones del VSESubjetivo, se aplicó el procedimiento de análisis de tablas contingencia con el estadístico chi-cuadrado de Pearson; utilizamos como una de las variables de contraste el Grupo (Clúster) de pertenencia y por otro lado la variable que tiene relación conceptual con la dimensión correspondiente de evaluación de VSE-Subjetivo.

\section{Resultados}

En este apartado ofrecemos los resultados del presente estudio, organizados de acuerdo con el siguiente esquema:

- En primer lugar, ofrecemos la solución para 4 conglomerados, obtenidos mediante el procedimiento de conglomerados (clústers) de $\mathrm{k}$ medias, para poder mostrar cómo son los perfiles de los sujetos (padres y madres que han respondido al cuestionario) de acuerdo con la escala de EEF-Historias (para más información ver Bisquert, 2017).

- En segundo lugar, ofrecemos los resultados del estudio de asociación, realizado mediante el procedimiento de análisis de tablas de contingencia, entre los estilos familiares determinados por el perfil de cada Grupo (clúster) y las variables que se relacionan con las dimensiones de evaluación de VSE-Subjetivo (Jornet et al., 2011; Sancho-Álvarez, Jornet y González-Such, 2017). Los resultados se organizan por las dimensiones de evaluación del VSE-Subjetivo.

- Después de cada análisis de tablas de contingencias se señalan los resultados más relevantes para nuestro estudio. Los resultados más significativos se debatirán en los apartados Discusión y Conclusiones.

A continuación, ofrecemos la Figura 1, en la que presentamos los perfiles de distribución de los cuatro grupos formados a partir del estudio de conglomerados (clústeres) para k-medias en función de las puntuaciones de la escala EEF-Historias (Estilo Autoritario, Estilo Integral, Estilo Sobreprotector, Estilo Permisivo, Estilo Democrático). 
Conglomerados de K Medias. Solución de 4 grupos.

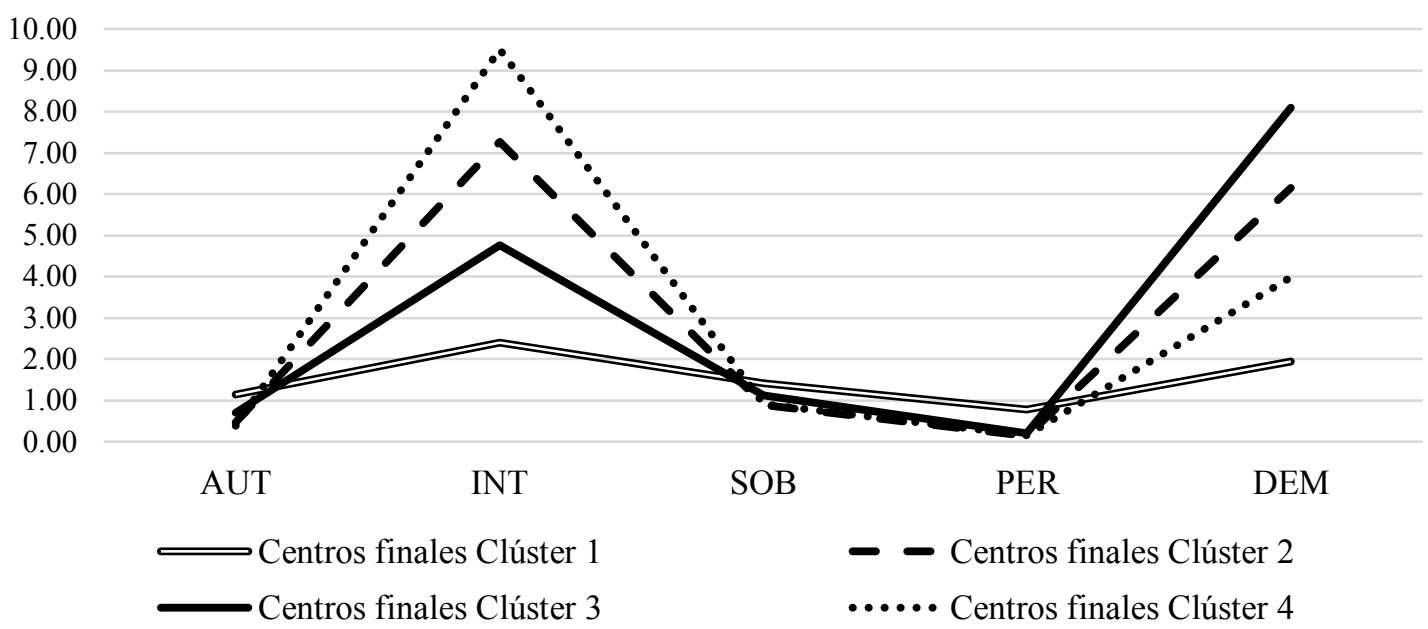

Figura 1. Centroides de solución final para el clúster de k medias para 4 grupos en la escala de EEF-Historias de Bisquert (2017).

Nota: Seleccionamos el gráfico de líneas porque nos permite diferenciar los perfiles más fácilmente, aunque el perfil no determina la existencia de puntuaciones en los puntos intermedios entre cada valor de la escala horizontal. Los códigos de la escala horizontal corresponden: AUT - Autoritario, INT - Integral, SOB Sobreprotector, PER - Permisivo, DEM - Democrático.

La tarea planteada a padres y madres se organizó en torno a historias que planteaban situaciones problemáticas. Para hacerles frente se ofrecían cinco alternativas (cada una correspondiente a un EEF), y se debía elegir una, la que mejor representaba el estilo propio. Aunque se ofrecían 15 historias, hay familias que no las han contestado todas. Padres y madres seleccionaron con mucha mayor frecuencia las alternativas vinculadas a los estilos Integral y Democrático, siendo mucho menos frecuentes los otros estilos. Eso se refleja en los perfiles de los grupos.

Así, a partir de la Figura 1, de acuerdo con la distribución de perfiles de cada grupo, vamos a caracterizar los perfiles como:

- Clúster 1: presenta niveles (puntuaciones) de los centroides muy bajas en todos los estilos, por lo que lo codificamos como "No-Def". Agrupa 261 casos que han dejado muchas historias sin completar, y no han mostrado preferencia por ningún EEF.

- Clúster 4: presenta el centroide más alto de los cuatro grupos para el estilo Integral (aproximadamente 9 puntos), y la tercera más alta puntuación del centroide en el estilo Democrático (aproximadamente 4 puntos), por lo que lo codificamos como "9Int4Dem". Es el grupo más numeroso, con 1790 casos, que se decantan fundamentalmente por el EEF Integral, y, en menor medida, el Democrático.

- Clúster 2: también presenta los centroides con puntuación bastante alta (segunda más alta) en los estilos Integral (7 puntos) y Democrático (6 puntos), por lo que le asignamos el código "7Int-6Dem". Agrupa 730 casos, que se decantan de forma equilibrada por los EEF Integral y Democrático.

- Clúster 3: presenta la puntuación más alta entre cuatro perfiles para el estilo Democrático (8 puntos) y $3^{a}$ puntuación más alta para el estilo Integral (4 puntos), así que le asignamos el código "8Dem-4Int". Agrupa 877 casos, que se decantan fundamentalmente por el EEF Democrático y, complementariamente, el Integral. 
Es de interés observar que los otros estilos educativos familiares (Sobreprotector, Permisivo y Autoritario) en general tienen una puntuación baja (no más de 2 puntos) como tendencia general para todos los respondientes.

La clasificación de los respondientes en estos 4 grupos, definidos por sus perfiles nos permite analizar la relación con las dimensiones de evaluación de valor social subjetivo de educación (VSESubjetivo), definidas por Jornet, Perales y Sánchez Delgado (2011) para estudiar si el EEF está vinculado con el valor social que en la familia otorgan a la educación como elemento diferencial del desarrollo personal y social de los individuos.

A continuación, ofrecemos los resultados de análisis de tablas de contingencias.

\subsection{Dimensión 1. Expectativas y metas educativas.}

En este apartado se plantea el análisis de la dimensión 1 de evaluación del VSE-Subjetivo a través de la variable del instrumento de evaluación de EEF: “¿Hasta qué nivel le gustaría como mínimo que estudie el niño/ la niña? Espera que al menos curse..." (código B09), con sus correspondientes niveles educativos que los padres señalan en función de sus expectativas y metas. En la Tabla 1 podemos observar cómo se distribuyen las tendencias para cuatro Grupos (clústeres).

Tabla 1.

Tabla de contingencias para las variables Grupo (clüster) y ¿Hasta qué nivel le gustaría como minimo que estudie el niño/ la niña? Espera que al menos curse... [B09].

\begin{tabular}{|c|c|c|c|c|c|c|c|c|c|}
\hline & $\mathrm{ESO}$ & Bach. & FP & Univ. & Decidirá & $\mathrm{NS} / \mathrm{NC}^{*}$ & Total \\
\hline \multirow{12}{*}{ 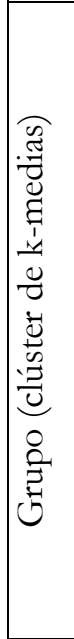 } & \multirow[t]{3}{*}{ No-Def } & Recuento & 15 & 9 & 10 & 138 & 49 & 24 & 245 \\
\hline & & $\%$ dentro de Grupo & $6,1 \%$ & $3,7 \%$ & $4,1 \%$ & $56,3 \%$ & $20,0 \%$ & $9,8 \%$ & $100,0 \%$ \\
\hline & & $\%$ dentro de [B09] & $13,0 \%$ & $4,7 \%$ & $5,4 \%$ & $5,7 \%$ & $10,6 \%$ & $12,6 \%$ & $6,9 \%$ \\
\hline & \multirow{3}{*}{$\begin{array}{l}7 \text { Int- } \\
6 \text { Dem }\end{array}$} & Recuento & 47 & 101 & 103 & 1208 & 210 & 90 & 1759 \\
\hline & & $\%$ dentro de Grupo & $2,7 \%$ & $5,7 \%$ & $5,9 \%$ & $68,7 \%$ & $11,9 \%$ & $5,1 \%$ & $100,0 \%$ \\
\hline & & $\%$ dentro de [B09] & $40,9 \%$ & $52,3 \%$ & $55,4 \%$ & $49,8 \%$ & $45,4 \%$ & $47,1 \%$ & $49,2 \%$ \\
\hline & \multirow{3}{*}{$\begin{array}{l}8 \mathrm{Dem}- \\
4 \operatorname{Int}\end{array}$} & Recuento & 32 & 42 & 35 & 451 & 114 & 38 & 712 \\
\hline & & $\%$ dentro de Grupo & $4,5 \%$ & $5,9 \%$ & $4,9 \%$ & $63,3 \%$ & $16,0 \%$ & $5,3 \%$ & $100,0 \%$ \\
\hline & & $\%$ dentro de [B09] & $27,8 \%$ & $21,8 \%$ & $18,8 \%$ & $18,6 \%$ & $24,6 \%$ & $19,9 \%$ & $19,9 \%$ \\
\hline & \multirow{3}{*}{$\begin{array}{l}9 \text { Int- } \\
4 \mathrm{Dem}\end{array}$} & Recuento & 21 & 41 & 38 & 627 & 90 & 39 & 856 \\
\hline & & $\%$ dentro de Grupo & $2,5 \%$ & $4,8 \%$ & $4,4 \%$ & $73,2 \%$ & $10,5 \%$ & $4,6 \%$ & $100,0 \%$ \\
\hline & & $\%$ dentro de [B09] & $18,3 \%$ & $21,2 \%$ & $20,4 \%$ & $25,9 \%$ & $19,4 \%$ & $20,4 \%$ & $24,0 \%$ \\
\hline \multirow{3}{*}{\multicolumn{2}{|c|}{ Total }} & Recuento & 115 & 193 & 186 & 2424 & 463 & 191 & 3572 \\
\hline & & $\%$ dentro de Grupo & $3,2 \%$ & $5,4 \%$ & $5,2 \%$ & $67,9 \%$ & $13,0 \%$ & $5,3 \%$ & $100,0 \%$ \\
\hline & & $\%$ dentro de [B09] & $100,0 \%$ & $100,0 \%$ & $100,0 \%$ & $100,0 \%$ & $100,0 \%$ & $100,0 \%$ & $100,0 \%$ \\
\hline
\end{tabular}

La Tabla 1 presenta la distribución de sujetos para las categorías de la variable B09 en función de cada Grupo (clúster). Las filas correspondientes a cada Grupo presentan datos de número de respondientes y los porcentajes dentro de B09 y también porcentajes dentro de Grupo. Para facilitar la lectura se han seleccionado las categorías de la B09 y sus porcentajes en itálica. Así, podemos ver que la ESO han escogido en total 115 sujetos, la categoría Bachillerato han escogido 193 sujetos, la FP 186 sujetos, la Universidad como expectativa han escogido 2424 padres y madres; por otro lado, dejan la decisión para sus hijos e hijas para cuando éstos sean mayores 463 padres y madres y 191 respondientes todavía no han pensado en ello.

Para facilitar la apreciación de la tendencia general, podemos observar esta misma distribución en la Figura 2 que agrupa las categorías de la B09 en el gráfico de barras para cada Grupo. 
Así, se observa la caracterización de cada Grupo (clúster) con los grupos formados por la variable B09.

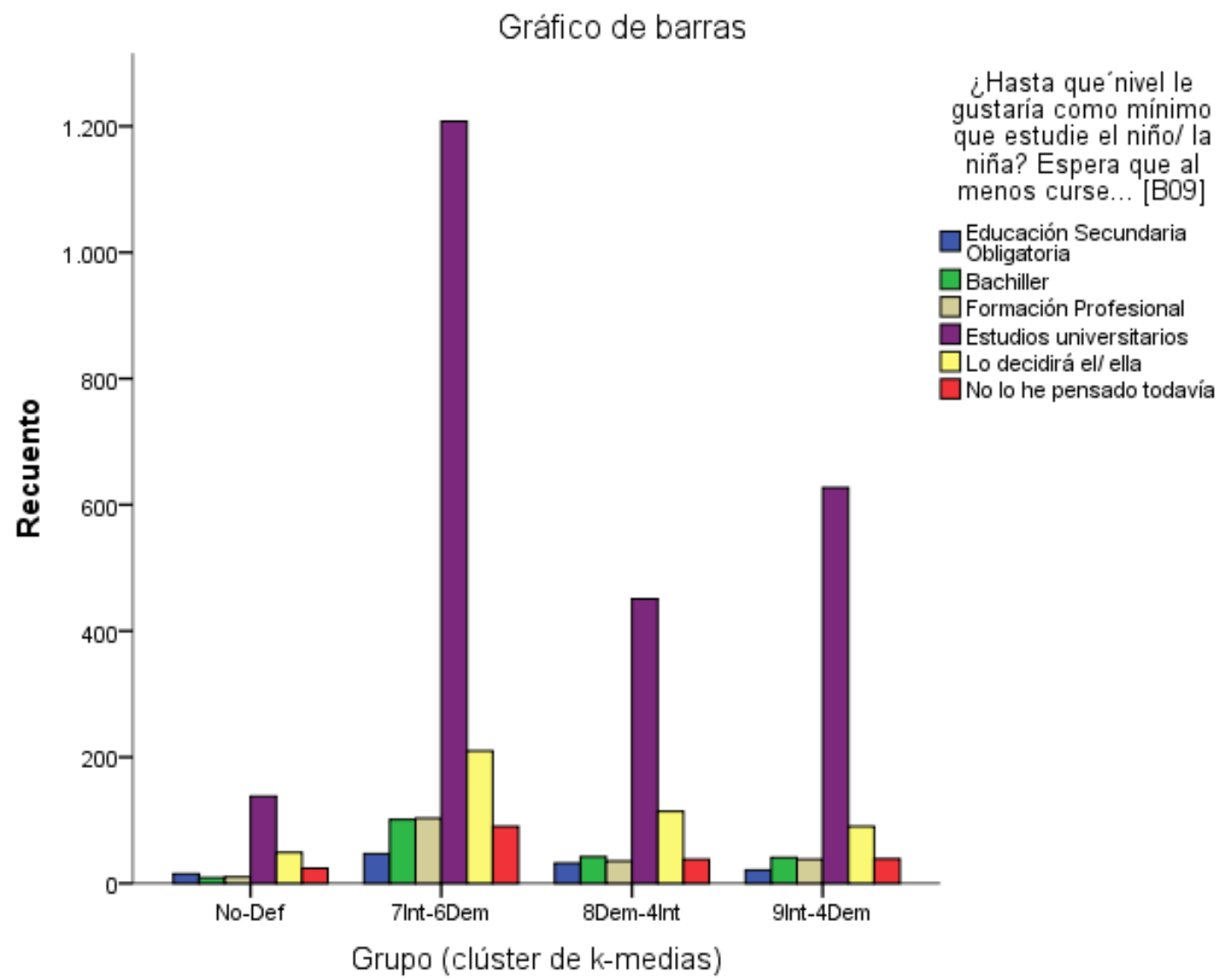

Figura 2. Gráfico de barras para la variable B09 en función de su pertenencia al Grupo (clúster).

De acuerdo con la Figura 2, el grueso de los respondientes, en todos los Grupos, seleccionan la categoría de Estudios Universitarios, seguida con mucho margen de la categoría "Lo decidirá él/ella". En la mayoría de los Grupos el nivel "ESO" es el que menos veces se ha seleccionado, salvo el caso del grupo "No-Def", donde los menos elegidos son el nivel "Bachiller" y el "FP". Además, de acuerdo con la Figura 2, podemos observar tanto la proporción de las categorías de la variable B09, distribuidas por Grupo (clúster), como la tendencia de distribución de cada categoría de la B09 por Grupos. Así, la tendencia a variar de la categoría de Estudios Universitarios muestra que es la categoría más elegida por grupo "9Int-4Dem" y "7Int-6Dem". Finalmente, sólo el 56,3\% de las familias del Grupo "No-Def" marca como expectativa los estudios universitarios, en contraste con otros Grupos, donde el porcentaje en esta categoría es más alto. En general, podríamos afirmar que los padres y las madres que tienen el perfil de estilo más Integral tienen más frecuentemente la expectativa de que sus hijos puedan estudiar en la Universidad.

A continuación, ofrecemos la Tabla 2 que contiene los estadísticos de la asociación entre ambas variables. 
Tabla 2.

Pruebas de chi-cuadrado

\begin{tabular}{lccc}
\hline & Valor & gl & Sig. asintótica (bilateral) \\
\hline Chi-cuadrado de Pearson & $59,830^{\text {a }}$ & 15 &, 000 \\
Razón de verosimilitudes & 55,657 & 15 &, 000 \\
Asociación lineal por lineal &, 447 & 1 &, 504 \\
N de casos válidos & 3572 & &
\end{tabular}

a. 0 casillas $(, 0 \%)$ tienen una frecuencia esperada inferior a 5. La frecuencia mínima esperada es 7,89.

Podemos confirmar, de acuerdo con la Tabla 2, que sí existe una relación estadísticamente significativa entre ambas variables. La razón de verosimilitudes es un estadístico asintóticamente equivalente a chi-cuadrado de Pearson y también presenta el nivel de significatividad asintótica menor que 0,05 , por lo que concluimos que existe asociación entre la pertenencia al Grupo (clúster) y el nivel de expectativa educativa para los hijos.

Por otro lado, el coeficiente de contingencia, el estadístico que indica el grado de asociación y varía de 0 a 1 , en el caso de asociación entre estas dos variables es igual a 0,128. Por lo tanto, podemos señalar que la asociación entre el EEF y el nivel de expectativa con la educación de los hijos es estadísticamente significativa pero muy baja. Debemos señalar que, al tratarse de las variables nominales, con estos estadísticos como mucho podemos calcular el grado de asociación que presentan las variables, pero no la dirección o naturaleza de tal asociación, que viene descrita por el análisis de las tablas de contingencia.

\subsection{Dimensión 2. Justicia Social y Educación.}

La Dimensión 2 de evaluación de Justicia Social y Educación se define como las consecuencias y/o beneficios perciben alumnado y familias que tiene la educación para el bienestar personal y social. En el caso de evaluación de EEF se relaciona con las tres variables que señalamos a continuación:

- “Con qué frecuencia suele hablar de las cosas del colegio con el niño/ con la niña?" [B07], con las categorías de respuesta: Nunca, Algunas veces, Bastantes veces, Muchas veces.

- “Está Usted satisfecho/a con los resultados de estudios del niño/ la niña?” [B10], con las categorías de respuesta: Nada satisfecho, Algo satisfecho, Bastante satisfecho y Muy satisfecho.

- “Está Usted satisfecho/a con el tiempo que le dedica al niño/ la niña?” [B11], con las mismas categorías de respuesta que la anterior.

A continuación, ofrecemos los resultados para el cálculo de asociación entre el Grupo (clúster) y las variables de la dimensión.

En todos los Grupos (clústeres) la distribución de las diferentes categorías de la variable B07 es aparentemente similar, tal y como podemos observar en la Figura 3. 


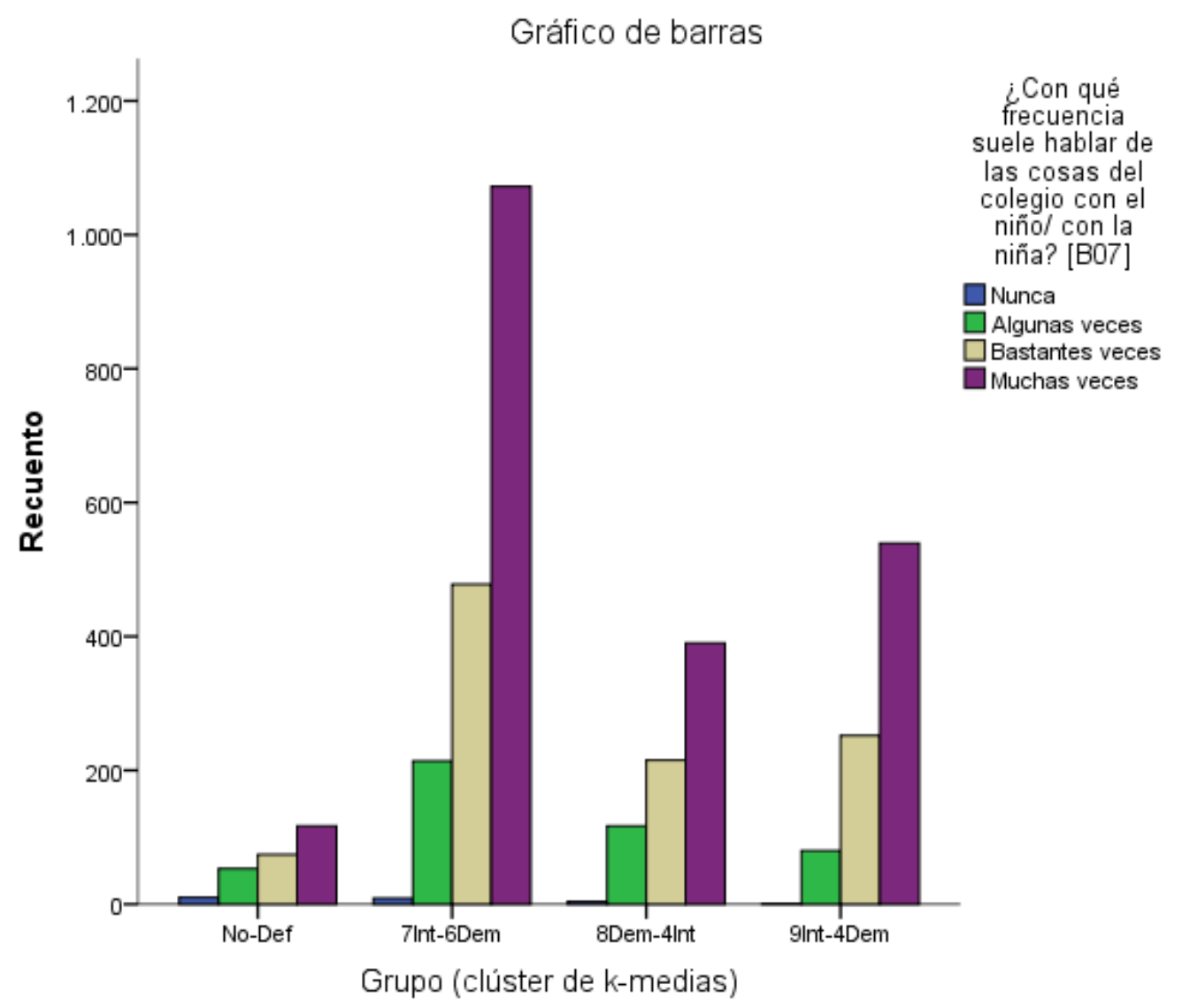

Figura 3. Gráfico de barras de distribución de la variable B07 en función del Grupo (clúster).

De acuerdo con la Figura 3, podemos observar que la proporción de la categoría "Muchas veces" en los grupos "7Int-6Dem" y "9Int-4Dem" es muy alta, en comparación con las otras categorías de la variable B07, aunque en otros Grupos el porcentaje de sujetos que marcan esta categoría también es alto; en los otros Grupos la proporción de los padres que declaran hablar muchas veces de las cosas del colegio con sus hijos es alta, pero no supera el doble del grupo que declara hablar bastantes veces con los hijos.

A continuación, podemos observar las distribuciones de una manera más exacta, con los datos de recuento y porcentajes de las categorías, en la Tabla 3.

Desde la Tabla 3 vemos que los padres que forman el Grupo "No-Def” son más autocríticos con la actitud con sus hijos: en comparación con otros Grupos (clústeres) aquí la categoría de aquellos que reconocen no hablar nunca con sus hijos es considerable (10 personas, 3,9\% de 254 personas en total que forman el Grupo). Si las mismas 10 personas del Grupo "No-Def" las consideramos como parte de la categoría de la variable B07 de sujetos que declaran no hablar nunca con sus hijos de las cosas del colegio (primera columna de datos, 24 personas), serían un 41,7\% del grupo, pese a que este grupo "No-Def" sólo incluye el 7\% del total de los sujetos. 
Tabla 3.

Tabla de contingencias para las variables Grupo (cluister) y ¿Con qué frecuencia suele hablar de las cosas del colegio con el niñol con la niña? [B07].

\begin{tabular}{|c|c|c|c|c|c|c|c|}
\hline & & & Nunca & $\begin{array}{l}\text { Algunas } \\
\text { veces }\end{array}$ & $\begin{array}{c}\text { Bastantes } \\
\text { veces }\end{array}$ & $\begin{array}{l}\text { Muchas } \\
\text { veces }\end{array}$ & Total \\
\hline Grupo (clúster & No-Def & Recuento & 10 & 53 & 74 & 117 & 254 \\
\hline & & $\%$ dentro de Grupo & $3,9 \%$ & $20,9 \%$ & $29,1 \%$ & $46,1 \%$ & $100,0 \%$ \\
\hline & & $\%$ dentro de [B07] & $41,7 \%$ & $11,4 \%$ & $7,3 \%$ & $5,5 \%$ & $7,0 \%$ \\
\hline & 7Int- & Recuento & 9 & 214 & 478 & 1072 & 1773 \\
\hline & 6Dem & $\%$ dentro de Grupo &, $5 \%$ & $12,1 \%$ & $27,0 \%$ & $60,5 \%$ & $100,0 \%$ \\
\hline & & $\%$ dentro de [B07] & $37,5 \%$ & $46,1 \%$ & $46,9 \%$ & $50,6 \%$ & $48,9 \%$ \\
\hline & 8Dem- & Recuento & 4 & 117 & 215 & 390 & 726 \\
\hline & $4 \ln t$ & $\%$ dentro de Grupo & $6 \%$ & $16,1 \%$ & $29,6 \%$ & $53,7 \%$ & $100,0 \%$ \\
\hline & & $\%$ dentro de [B07] & $16,7 \%$ & $25,2 \%$ & $21,1 \%$ & $18,4 \%$ & $20,0 \%$ \\
\hline & 9Int- & Recuento & 1 & 80 & 252 & 539 & 872 \\
\hline & 4Dem & $\%$ dentro de Grupo &, $1 \%$ & $9,2 \%$ & $28,9 \%$ & $61,8 \%$ & $100,0 \%$ \\
\hline & & $\%$ dentro de [B07] & $4,2 \%$ & $17,2 \%$ & $24,7 \%$ & $25,4 \%$ & $24,1 \%$ \\
\hline Total & & Recuento & 24 & 464 & 1019 & 2118 & 3625 \\
\hline & & $\%$ dentro de Grupo &, $7 \%$ & $12,8 \%$ & $28,1 \%$ & $58,4 \%$ & $100,0 \%$ \\
\hline & & $\%$ dentro de [B07] & $100,0 \%$ & $100,0 \%$ & $100,0 \%$ & $100,0 \%$ & $100,0 \%$ \\
\hline
\end{tabular}

A continuación, se ofrecen los resultados de la prueba chi-cuadrado para el análisis de la asociación entre Grupos y la variable B07 (Tabla 4).

Tabla 4.

Pruebas de chi-cuadrado

\begin{tabular}{llll}
\hline & Valor & gl & Sig. asintótica (bilateral) \\
\hline Chi-cuadrado de Pearson & $88,781^{\text {a }}$ & 9 &, 000 \\
Razón de verosimilitudes & 67,808 & 9 &, 000 \\
Asociación lineal por lineal & 13,899 & 1 &, 000 \\
N de casos válidos & 3625 & & \\
\hline
\end{tabular}

a. 2 casillas $(12,5 \%)$ tienen una frecuencia esperada inferior a 5. La frecuencia mínima esperada es 1,68 .

Los resultados de cálculo de asociación entre las variables confirman la existencia de la asociación estadísticamente significativa entre ambas variables. En este caso, el coeficiente de contingencia es de 0,155 . Por lo que podemos afirmar que, aunque la asociación es estadísticamente significativa, el nivel de esta asociación es muy bajo.

De otra manera diríamos que se confirma la relación entre el estilo educativo familiar prioritario y el tiempo que se dedica a hablar con los hijos sobre las cosas del colegio.

A continuación, ofrecemos los resultados de análisis entre la relación de Grupos (clústeres) y la variable “¿Está Usted satisfecho/a con los resultados de estudios del niño/la niña?” (B10). 


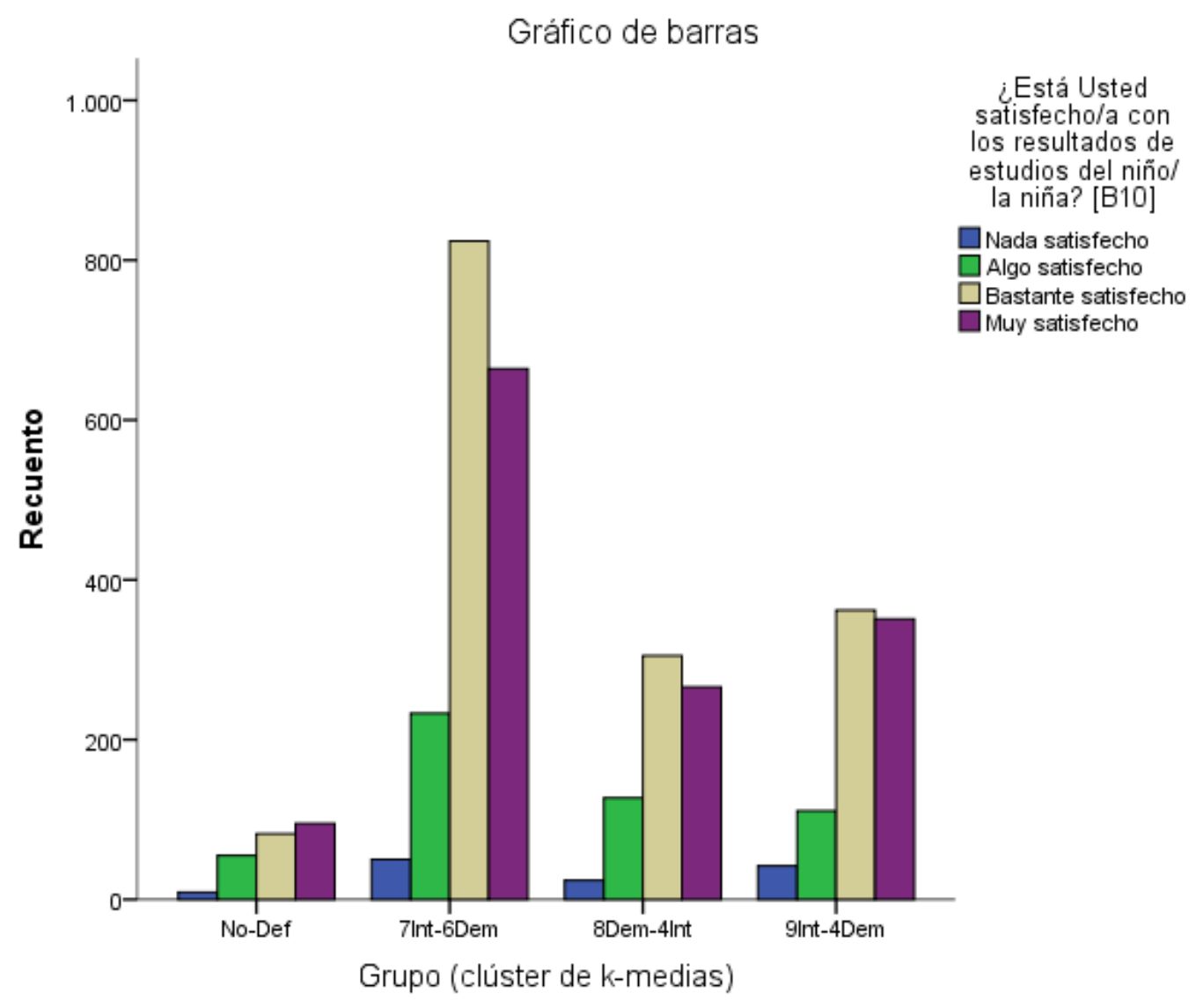

Figura 4. Gráfico de barras para la variable B10 en función de su distribución por Grupos (clústeres).

El gráfico de barras, presentado en la Figura 4, muestra una distribución desigual entre las categorías de padres que valoran la satisfacción con los resultados de sus hijos por los Grupos correspondientes a estilos familiares marcados. En este caso, en contraste con la anterior variable (dedicación de tiempo para hablar de cosas del colegio con los hijos), los padres son más críticos con la valoración: la categoría "Muy satisfecho" no es la dominante, sino la de "Bastante satisfecho". También se puede apreciar en comparación a otras categorías de la variable B10, un número significativo de padres que no están nada satisfechos con los resultados escolares de sus hijos.

Para concretar los números de respondientes en cada categoría y sus porcentajes por Grupo y categoría de la B10, recurrimos a los datos presentados en la Tabla 5.

Desde la Tabla 5 podemos observar que los padres que están menos satisfechos con los resultados escolares de sus hijos son los padres que no tienen un EEF marcado (Grupo No-Def), pues más de un $26 \%$ de ellos se manifiestan "Nada" o "Algo satisfechos". Frente a ellos, los padres de los grupos "7Int-6Dem" y "9Int-4Dem" son los que se muestran más satisfechos, sumando más del $80 \%$ en cada caso los que manifiestan estar "Bastante" y "Muy satisfechos" con los resultados escolares. Balanceando esta información, se puede destacar que dentro del Cluster "No-Def" hay un grupo significativo de padres que dice estar "Muy satisfecho" (aproximadamente un 40\%, cercano al porcentaje del grupo más alto "9Int-4Dem"). Podemos decir que la tónica general es bastante moderada, en contraste con la valoración de la dedicación de los padres a hablar con sus hijos sobre las tareas escolares, que mostró gran satisfacción de los padres con su propia aportación. 
Tabla 5.

Tabla de contingencias para las variables Grupo (clüster) y ¿Está Usted satisfecho/ a con los resultados de estudios del niño/ la niña? [B10].

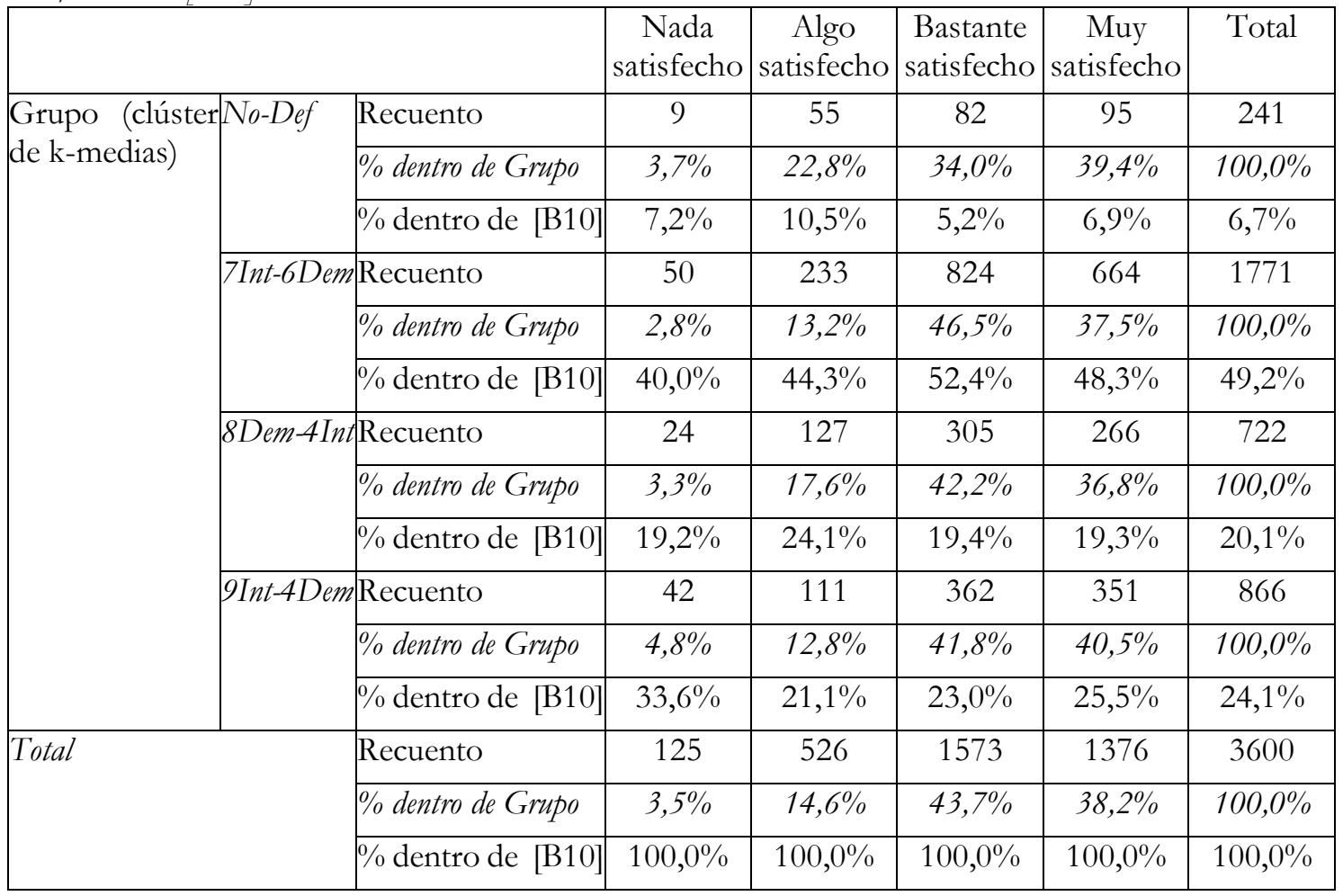

Para contrastar si existe la asociación estadística entre ambas variables, vemos la Tabla 6, a continuación.

Tabla 6.

Pruebas de chi-cuadrado.

\begin{tabular}{llll}
\hline & Valor & gl & Sig. asintótica (bilateral) \\
\hline Chi-cuadrado de Pearson & $38,323^{\mathrm{a}}$ & 9 &, 000 \\
Razón de verosimilitudes & 36,562 & 9 &, 000 \\
Asociación lineal por lineal &, 075 & 1 &, 784 \\
N de casos válidos & 3600 & &
\end{tabular}

a. 0 casillas $(, 0 \%)$ tienen una frecuencia esperada inferior a 5. La frecuencia mínima esperada es 8,37.

De acuerdo con los datos de la Tabla 6, podemos afirmar la existencia de una relación estadísticamente significativa entre los estilos educativos familiares con los que predominantemente se identifican padres y madres y su satisfacción/insatisfacción con los resultados escolares de sus hijos. El coeficiente de contingencia para este caso es de 0,103 , por lo que podemos decir que la asociación es estadísticamente significativa, pero muy baja.

A continuación, se ofrecen los resultados y comentarios para el caso de estudio de la relación entre los Grupos (clústeres) y la variable "¿Está Usted satisfecho con el tiempo que le dedica al niño/ la niña?” (B11). 


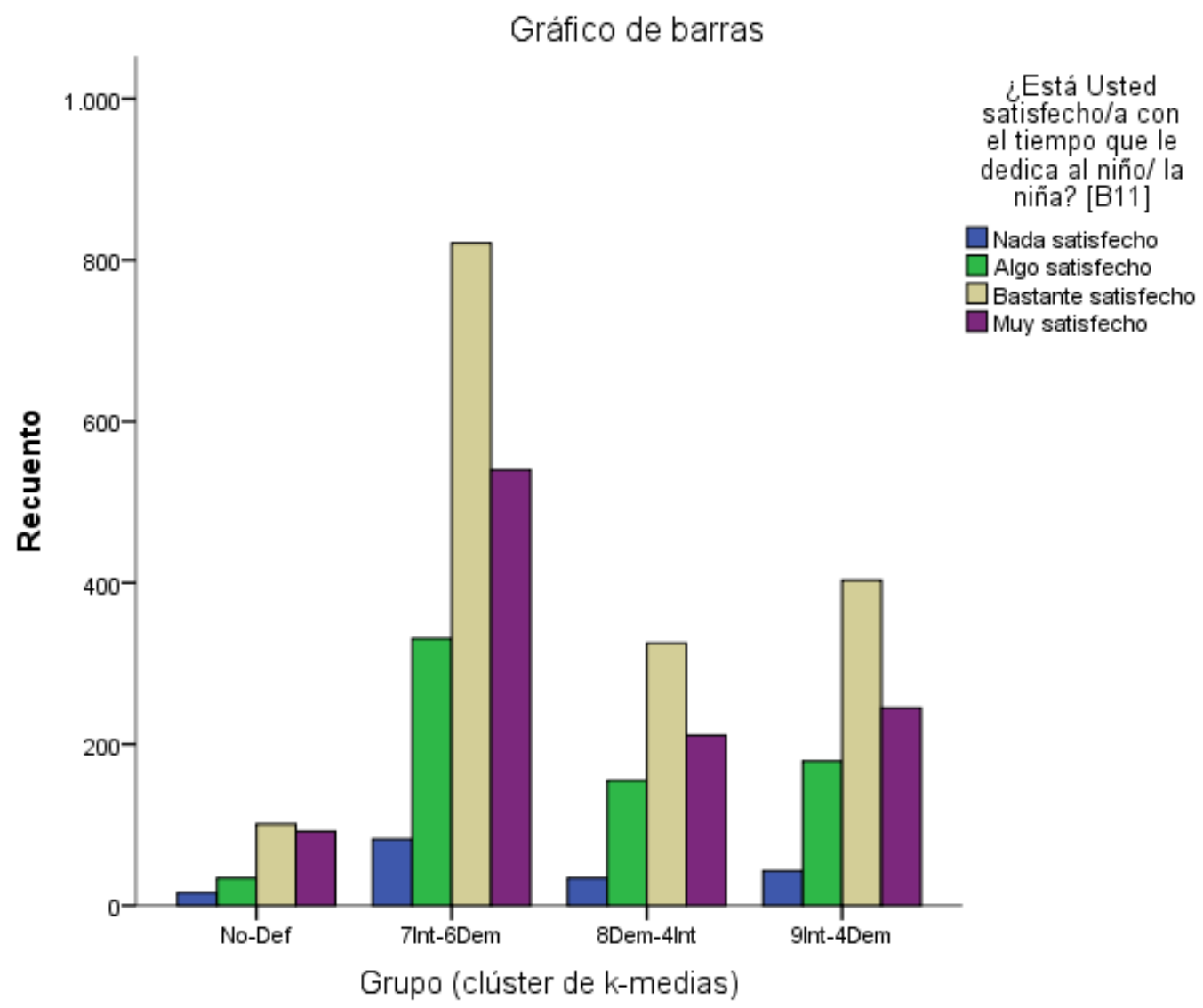

Figura 5. Gráfico de barras para la variable B11 en función de su pertenencia al Grupo (clúster).

De acuerdo con el gráfico de barras representado en la Figura 5, podemos observar que todas las categorías de la variable B11 tienen una tendencia similar de distribución de los porcentajes en diferentes Grupos (clústeres), aunque en el caso del Grupo "No-Def” la distancia entre el número de sujetos que conforman las categorías "Bastante satisfecho" y "Muy satisfecho" es muy pequeña. La categoría predominante de respuesta es la primera, seguida de la segunda.

Podemos concretar los datos de una manera más exacta en la Tabla 7.

De acuerdo con la Tabla 7, en general, los padres están satisfechos y muy satisfechos con el tiempo dedicado a sus hijos, predominando la categoría de bastante satisfechos, tal y como comentamos anteriormente en la Figura 5. Los padres, de todos los EEF, se muestran sólo moderadamente satisfechos con el tiempo que les dedican a los niños. 
Tabla 7.

Tabla de contingencias para las variables Grupo (clüster) y ¿Está Usted satisfecho/a con el tiempo que le dedica al niño/ la niña? [B11].

\begin{tabular}{|c|c|c|c|c|c|c|c|}
\hline & & & $\begin{array}{c}\text { Nada } \\
\text { satisfecho }\end{array}$ & \begin{tabular}{|c|} 
Algo \\
satisfecho
\end{tabular} & $\begin{array}{c}\text { Bastante } \\
\text { satisfecho }\end{array}$ & $\begin{array}{c}\text { Muy } \\
\text { satisfecho }\end{array}$ & Total \\
\hline \multirow{12}{*}{$\begin{array}{l}\text { Grupo (clúster } 1 \\
\text { de k-medias) }\end{array}$} & No-Def & Recuento & 16 & 34 & 101 & 92 & 243 \\
\hline & & $\%$ dentro de Grupo & $6,6 \%$ & $14,0 \%$ & $41,6 \%$ & $37,9 \%$ & $100,0 \%$ \\
\hline & & $\%$ dentro de [B11] & $9,1 \%$ & $4,9 \%$ & $6,1 \%$ & $8,5 \%$ & $6,7 \%$ \\
\hline & 7Int-6Dem & Recuento & 82 & 331 & 821 & 540 & 1774 \\
\hline & & $\%$ dentro de Grupo & $4,6 \%$ & $18,7 \%$ & $46,3 \%$ & $30,4 \%$ & $100,0 \%$ \\
\hline & & $\%$ dentro de [B11] & $46,9 \%$ & $47,4 \%$ & $49,8 \%$ & $49,6 \%$ & $49,1 \%$ \\
\hline & $8 D e m-4 I n$ & Recuento & 34 & 155 & 325 & 211 & 725 \\
\hline & & $\%$ dentro de Grupo & $4,7 \%$ & $21,4 \%$ & $44,8 \%$ & $29,1 \%$ & $100,0 \%$ \\
\hline & & $\%$ dentro de [B11] & $19,4 \%$ & $22,2 \%$ & $19,7 \%$ & $19,4 \%$ & $20,1 \%$ \\
\hline & 9Int-4Dem & Recuento & 43 & 179 & 403 & 245 & 870 \\
\hline & & $\%$ dentro de Grupo & $4,9 \%$ & $20,6 \%$ & $46,3 \%$ & $28,2 \%$ & $100,0 \%$ \\
\hline & & $\%$ dentro de [B11] & $24,6 \%$ & $25,6 \%$ & $24,4 \%$ & $22,5 \%$ & $24,1 \%$ \\
\hline \multirow{3}{*}{\multicolumn{2}{|c|}{ Total }} & Recuento & 175 & 699 & 1650 & 1088 & 3612 \\
\hline & & $\%$ dentro de Grupo & $4,8 \%$ & $19,4 \%$ & $45,7 \%$ & $30,1 \%$ & $100,0 \%$ \\
\hline & & $\%$ dentro de [B11] & $100,0 \%$ & $100,0 \%$ & $100,0 \%$ & $100,0 \%$ & $100,0 \%$ \\
\hline
\end{tabular}

A continuación, se ofrecen estadísticos de contraste para el análisis de asociación entre ambas variables mencionadas.

Tabla 8.

Pruebas de chi-cuadrado.

\begin{tabular}{llll}
\hline & Valor & gl & Sig. asintótica (bilateral) \\
\hline Chi-cuadrado de Pearson & $15,498^{\mathrm{a}}$ & 9 &, 078 \\
Razón de verosimilitudes & 15,364 & 9 &, 081 \\
Asociación lineal por lineal & 4,903 & 1 &, 027 \\
N de casos válidos & 3612 & &
\end{tabular}

a. 0 casillas $(, 0 \%)$ tienen una frecuencia esperada inferior a 5 . La frecuencia mínima esperada es 11,77.

De acuerdo con los datos de la Tabla 8, vemos que se confirma la hipótesis de independencia para las variables de estudio. El coeficiente de contingencia para este caso es de 0,065, que también muestra una relación nula entre los datos. Podemos afirmar que no se identifica la asociación estadística entre el EEF y el nivel de satisfacción con el tiempo pasado con los hijos.

\subsection{Dimensión 3. Valor diferencial de la educación.}

La presente dimensión se define como el valor intrínseco para la vida que dan las familias a la educación. La variable que corresponde en el estudio realizado sobre EEF es la de "Le enseño a asumir la responsabilidad de sus actuaciones" [B31]; podemos ver la representación gráfica de la distribución de sus opciones de respuesta (Nunca, Alguna vez, Habitualmente, Siempre) en función de su pertenencia al Grupo (clúster) en la Figura 6, a continuación. 


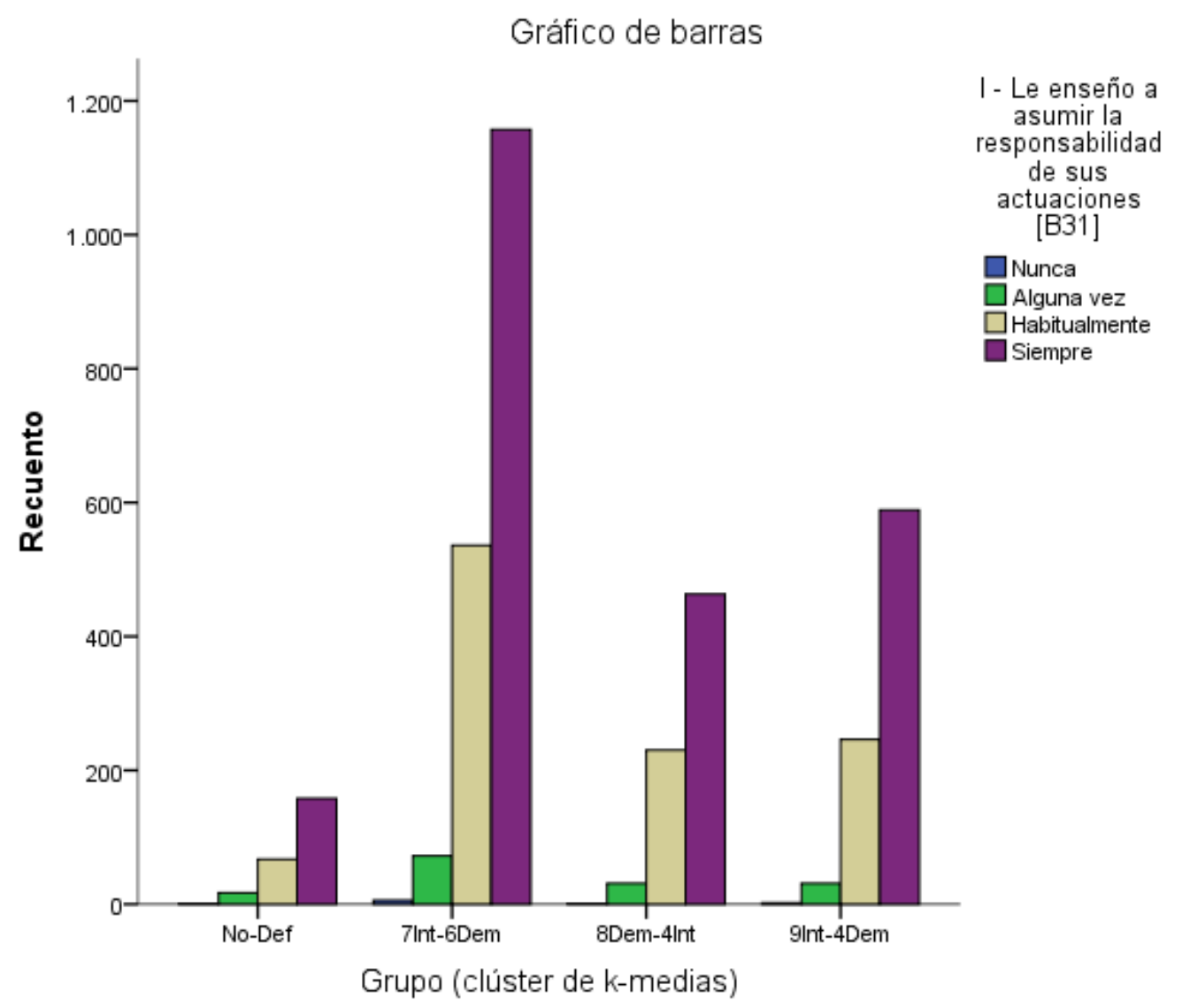

Figura 6. Gráfico de barras para la variable B31 en función de su pertenencia al Grupo (clúster).

El gráfico de barras presente en la Figura 6 nos muestra la distribución aparentemente homogénea de las categorías de la B31 por los perfiles de EEF representados en Grupos. Los padres que declaran "Siempre" y "Habitualmente" enseñar a asumir la responsabilidad por sus acciones a sus hijos forman parte del grueso de los grupos, además, las proporciones de diferentes categorías de la B31 son aparentemente homogéneas en cada Grupo.

A continuación, en la Tabla 9 podemos observar los datos exactos de número de sujetos y porcentajes para grupos formados por los perfiles de clústeres y por la B31.

La Tabla 9 confirma una tendencia homogénea en distribución de las respuestas a la cuestión sobre la enseñanza de la responsabilidad por parte de los padres y madres en diferentes Grupos formados por clústeres, es decir, el estilo educativo parece no marcar diferencia en cuanto a la intención de los padres en cuanto a la enseñanza de la responsabilidad sobre las acciones de sus hijos. 
Tabla 9.

Tabla de contingencias para las variables Grupo (clúster) y Le enseño a asumir la responsabilidad de sus actuaciones B317.

\begin{tabular}{|c|c|c|c|c|c|c|c|}
\hline & & & Nunca & $\begin{array}{l}\text { Alguna } \\
\text { vez }\end{array}$ & $\begin{array}{l}\text { Habitual } \\
\text { mente }\end{array}$ & Siempre & Total \\
\hline \multirow{12}{*}{$\begin{array}{l}\text { Grupo (clúster } 1 \\
\text { de k-medias) }\end{array}$} & \multirow{3}{*}{ No-Def } & Recuento & 1 & 17 & 67 & 158 & 243 \\
\hline & & $\%$ dentro de Grupo & $4 \%$ & $7,0 \%$ & $27,6 \%$ & $65,0 \%$ & $100,0 \%$ \\
\hline & & $\%$ dentro de [B31] & $10,0 \%$ & $11,3 \%$ & $6,2 \%$ & $6,7 \%$ & $6,7 \%$ \\
\hline & \multirow{3}{*}{$\begin{array}{l}\text { 7Int- } \\
\text { 6Dem }\end{array}$} & Recuento & 6 & 72 & 536 & 1157 & 1771 \\
\hline & & $\%$ dentro de Grupo &, $3 \%$ & $4,1 \%$ & $30,3 \%$ & $65,3 \%$ & $100,0 \%$ \\
\hline & & $\%$ dentro de [B31] & $60,0 \%$ & $47,7 \%$ & $49,7 \%$ & $48,9 \%$ & $49,1 \%$ \\
\hline & \multirow{3}{*}{$\begin{array}{l}\text { 8Dem- } \\
4 \text { Int }\end{array}$} & Recuento & 1 & 31 & 230 & 463 & 725 \\
\hline & & $\%$ dentro de Grupo &, $1 \%$ & $4,3 \%$ & $31,7 \%$ & $63,9 \%$ & $100,0 \%$ \\
\hline & & $\%$ dentro de [B31] & $10,0 \%$ & $20,5 \%$ & $21,3 \%$ & $19,6 \%$ & $20,1 \%$ \\
\hline & \multirow{3}{*}{$\begin{array}{l}\text { 9Int- } \\
\text { 4Dem }\end{array}$} & Recuento & 2 & 31 & 246 & 589 & 868 \\
\hline & & $\%$ dentro de Grupo & $2 \%$ & $3,6 \%$ & $28,3 \%$ & $67,9 \%$ & $100,0 \%$ \\
\hline & & $\%$ dentro de [B31] & $20,0 \%$ & $20,5 \%$ & $22,8 \%$ & $24,9 \%$ & $24,1 \%$ \\
\hline \multirow{3}{*}{\multicolumn{2}{|c|}{ Total }} & Recuento & 10 & 151 & 1079 & 2367 & 3607 \\
\hline & & $\%$ dentro de Grupo &, $3 \%$ & $4,2 \%$ & $29,9 \%$ & $65,6 \%$ & $100,0 \%$ \\
\hline & & $\%$ dentro de [B31] & $100,0 \%$ & $100,0 \%$ & $100,0 \%$ & $100,0 \%$ & $100,0 \%$ \\
\hline
\end{tabular}

A continuación, podemos comprobar en la Tabla 10 los estadísticos de contraste chicuadrado en cuanto a la relación entre las dos variables mencionadas.

Tabla 10.

Pruebas de chi-cuadrado.

\begin{tabular}{llll}
\hline & Valor & gl & Sig. asintótica (bilateral) \\
\hline Chi-cuadrado de Pearson & $9,488^{\mathrm{a}}$ & 9 &, 394 \\
Razón de verosimilitudes & 8,841 & 9 &, 452 \\
Asociación lineal por lineal & 2,272 & 1 &, 132 \\
N de casos válidos & 3607 & &
\end{tabular}

a. 4 casillas $(25,0 \%)$ tienen una frecuencia esperada inferior a 5. La frecuencia mínima esperada es , 67.

En el caso de las variables "Le enseño a asumir la responsabilidad de sus actuaciones" y el Grupo, formado por los perfiles de estilos educativos familiares no se demuestra la relación estadísticamente significativa entre ambas. El coeficiente de contingencia para este caso es de 0,051, lo que también aporta el nivel de relación nula.

\subsection{Dimensión 4. Obstáculos y facilitadores}

La dimensión 4 de evaluación del VSE-Subjetivo se refleja en la percepción de los elementos que pueden ayudar o dificultar el proceso educativo. En nuestro caso está representado por la variable "Teniendo en cuenta sus circunstancias actuales, ¿Quién cree Usted que tiene, fundamentalmente, la responsabilidad de la educación del niño/ de la niña? [B03], con sus opciones de respuesta: La familia, 
El Centro Escolar, Es una responsabilidad compartida por igual entre la familia y el Centro Escolar, Es una responsabilidad del niño/ de la niña, y Otros. En la Figura 7 podemos visualizar el gráfico de barras de distribución de las respuestas en función de las categorías de la B03 por Grupos formados por los perfiles de EEF basados en el estudio de clústeres.

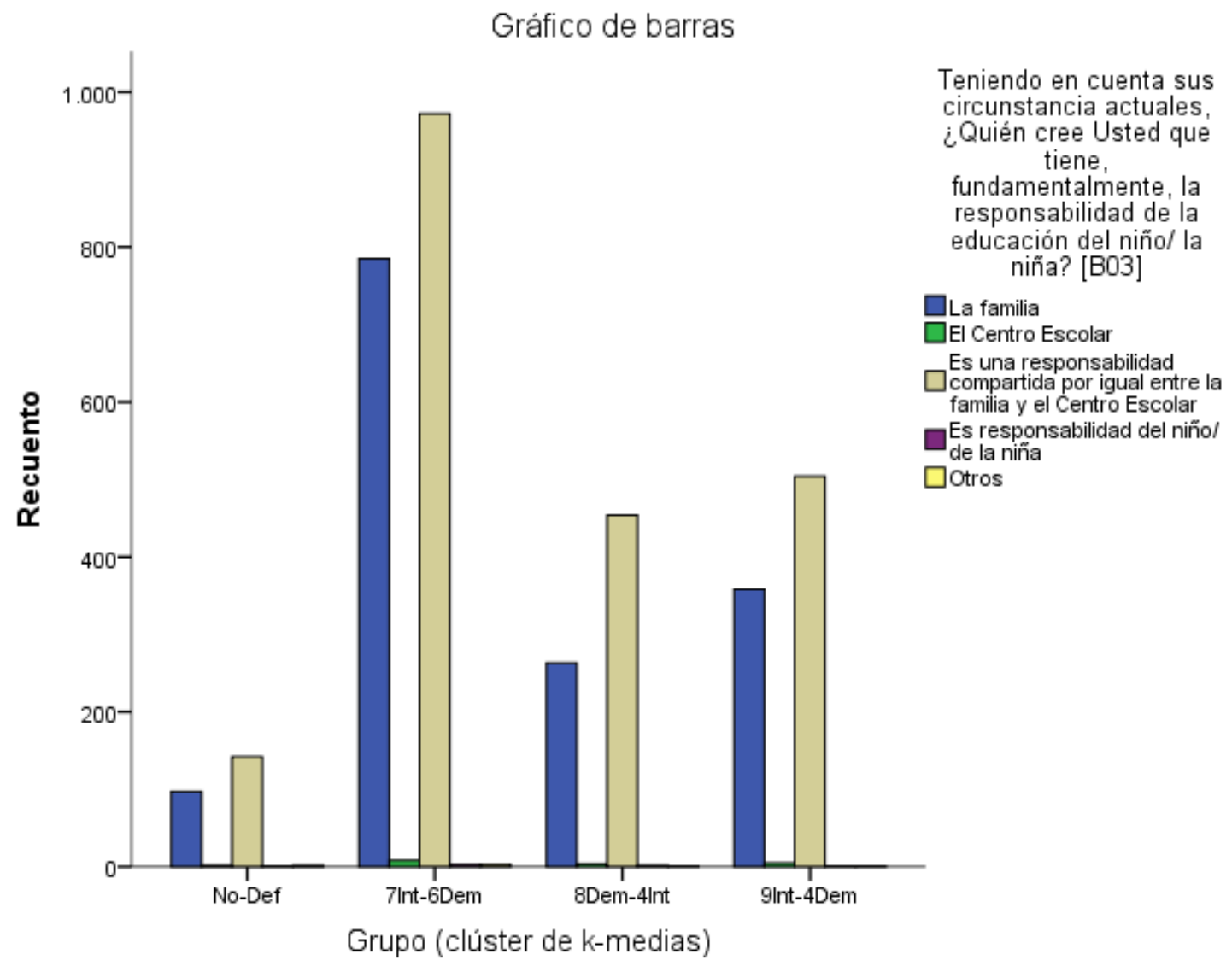

Figura 7. Gráfico de barras para la variable B03 en función de su pertenencia al Grupo (clúster).

De acuerdo con el gráfico (Figura 7) podemos apreciar que las respuestas se concentran en "La Familia" y "La Escuela", y que, aparentemente, no hay diferencias en la distribución de las categorías de la B03 por los Grupos, ni tampoco hay diferencias en las proporciones de la B03 por cada Grupo. En otras palabras, la tendencia de distribución no parece indicar que haya diferencias significativas marcadas por el estilo educativo en cuanto a la valoración de la responsabilidad sobre la educación de los hijos.

En la Tabla 11, a continuación, se pueden observar las distribuciones más exactas de los sujetos en función de ambas variables.

De acuerdo con la distribución de las categorías de la Tabla 11 podemos afirmar que la tendencia general de responder es muy homogénea en todos los Grupos, por lo que no podemos destacar las diferencias que podrían ser marcadas por alguna de las categorías de ninguna de las dos variables. 
Tabla 11.

Tabla de contingencias para las variables Grupo (cluster) y Teniendo en cuenta sus circunstancias actuales, ¿Quién cree Usted que tiene, fundamentalmente, la responsabilidad de la educación del niño/ la niña? [B03]

\begin{tabular}{|c|c|c|c|c|c|c|c|c|}
\hline & & & Familia & $\begin{array}{l}\text { Centro } \\
\text { Escolar }\end{array}$ & $\begin{array}{c}\text { Comparti } \\
\text { da Fam- } \\
\text { Esc. }\end{array}$ & $\begin{array}{c}\text { Del } \\
\text { niño/la } \\
\text { niña }\end{array}$ & Otros & Total \\
\hline \multirow{12}{*}{$\begin{array}{l}\text { Grupo } \\
\text { (clúster de k- } \\
\text { medias) }\end{array}$} & \multirow{3}{*}{ No-Def } & Recuento & 97 & 2 & 142 & 1 & 2 & 244 \\
\hline & & $\%$ dentro de Grupo & $39,8 \%$ &, $8 \%$ & $58,2 \%$ & ,4\% &, $8 \%$ & $100,0 \%$ \\
\hline & & $\%$ dentro de $[\mathrm{B} 03$ & $6,5 \%$ & $10,5 \%$ & $6,9 \%$ & $14,3 \%$ & $28,6 \%$ & $6,8 \%$ \\
\hline & \multirow{3}{*}{$\begin{array}{l}7 \text { Int- } \\
6 \mathrm{Dem}\end{array}$} & Recuento & 785 & 8 & 972 & 3 & 3 & 1771 \\
\hline & & $\%$ dentro de Grupo & $44,3 \%$ &, $5 \%$ & $54,9 \%$ &, $2 \%$ & $2 \%$ & $100,0 \%$ \\
\hline & & $\%$ dentro de $[\mathrm{B} 03$ & $52,2 \%$ & $42,1 \%$ & $46,9 \%$ & $42,9 \%$ & $42,9 \%$ & $49,1 \%$ \\
\hline & \multirow{3}{*}{$\begin{array}{l}8 D e m- \\
4 I n t\end{array}$} & Recuento & 263 & 4 & 454 & 2 & 1 & 724 \\
\hline & & $\%$ dentro de Grupo & $36,3 \%$ &, $6 \%$ & $62,7 \%$ & $3 \%$ &, $1 \%$ & $100,0 \%$ \\
\hline & & $\%$ dentro de $[\mathrm{B} 03]$ & $17,5 \%$ & $21,1 \%$ & $21,9 \%$ & $28,6 \%$ & $14,3 \%$ & $20,1 \%$ \\
\hline & \multirow{3}{*}{$\begin{array}{l}\text { 9Int- } \\
4 \mathrm{Dem}\end{array}$} & Recuento & 358 & 5 & 504 & 1 & 1 & 869 \\
\hline & & $\%$ dentro de Grupo & $41,2 \%$ &, $6 \%$ & $58,0 \%$ & , 1\% & ,1\% & $100,0 \%$ \\
\hline & & $\%$ dentro de $[\mathrm{B} 03$ & $23,8 \%$ & $26,3 \%$ & $24,3 \%$ & $14,3 \%$ & $14,3 \%$ & $24,1 \%$ \\
\hline \multirow{3}{*}{\multicolumn{2}{|c|}{ Total }} & Recuento & 1503 & 19 & 2072 & 7 & 7 & 3608 \\
\hline & & $\%$ dentro de Grupo & $41,7 \%$ &, $5 \%$ & $57,4 \%$ & $2 \%$ & $2 \%$ & $100,0 \%$ \\
\hline & & $\%$ dentro de $[\mathrm{B} 03$ & $100,0 \%$ & $100,0 \%$ & $100,0 \%$ & $100,0 \%$ & $100,0 \%$ & $100,0 \%$ \\
\hline
\end{tabular}

A continuación, para confirmar la idea podemos observar la Tabla 12 que presenta estadísticos para el estudio chi-cuadrado de la relación entre ambas variables.

Tabla 12.

Pruebas de chi-cuadrado.

\begin{tabular}{llll}
\hline & Valor & gl & Sig. asintótica (bilateral) \\
\hline Chi-cuadrado de Pearson & $20,987^{\mathrm{a}}$ & 12 &, 051 \\
Razón de verosimilitudes & 18,760 & 12 &, 094 \\
Asociación lineal por lineal & 1,882 & 1 &, 170 \\
N de casos válidos & 3608 & & \\
\hline
\end{tabular}

a. 11 casillas $(55,0 \%)$ tienen una frecuencia esperada inferior a 5 . La frecuencia mínima esperada es , 47.

La Tabla 12 muestra que la hipótesis de independencia de ambas variables es cierta. El coeficiente de contingencia para este caso es de 0,076, lo que muestra la relación nula entre ambas variables. En otras palabras, no se puede demostrar la relación estadística entre el EEF la opinión en cuanto a la responsabilidad de la educación del hijo.

En resumen, podemos afirmar que existe la relación estadísticamente significativa para los perfiles predominantes en la educación familiar y algunas de las variables que fueron objeto de nuestro estudio y presentadas a continuación en la Tabla 13. 
Tabla 13.

Resumen del estudio de relación entre las variables

\begin{tabular}{|c|c|c|c|c|}
\hline $\begin{array}{l}\text { Dimensión de } \\
\text { evaluación de } \\
\text { VSE-Subjetivo }\end{array}$ & Variable EEF & $\begin{array}{c}\text { Relación } \\
\text { significativa } \\
\text { con los estilos* }\end{array}$ & $\begin{array}{l}\text { Valor del } \\
\text { coef. } \\
\text { contingencia }\end{array}$ & $\begin{array}{l}\text { Nivel de } \\
\text { relación }\end{array}$ \\
\hline $\begin{array}{l}\text { 1. Expectativas } \\
\text { y metas } \\
\text { educativas. }\end{array}$ & $\begin{array}{l}\text { ¿Hasta qué nivel le gustaría } \\
\text { como mínimo que estudie el } \\
\text { niño/ la niña? Espera que al } \\
\text { menos curse...” (B09) }\end{array}$ & Sí & 0,128 & Baja \\
\hline \multirow[t]{3}{*}{$\begin{array}{l}\text { 2. Justicia } \\
\text { Social y } \\
\text { Educación. }\end{array}$} & $\begin{array}{l}\text { ¿Con qué frecuencia suele } \\
\text { hablar de las cosas del colegio } \\
\text { con el niño/ con la niña?" } \\
\text { (B07) }\end{array}$ & Sí & 0,155 & Baja \\
\hline & $\begin{array}{l}\text { ¿Está Usted satisfecho/a con } \\
\text { los resultados de estudios del } \\
\text { niño/ la niña?" (B10) }\end{array}$ & Sí & 0,103 & Baja \\
\hline & $\begin{array}{l}\text { ¿Está Usted satisfecho/a con } \\
\text { el tiempo que le dedica al } \\
\text { niño/ la niña? (B11) }\end{array}$ & No & 0,065 & Nula \\
\hline $\begin{array}{l}\text { 3. Valor } \\
\text { diferencial de } \\
\text { la educación. }\end{array}$ & $\begin{array}{lll}\text { Le enseño a } & \text { asumir } & \text { la } \\
\text { responsabilidad } & \text { de } & \text { sus } \\
\text { actuaciones (B31) } & & \end{array}$ & No & 0,051 & Nula \\
\hline $\begin{array}{l}\text { 4. Obstáculos } \\
\text { y facilitadores. }\end{array}$ & $\begin{array}{l}\text { ¿Quién cree Usted que tiene, } \\
\text { fundamentalmente, la } \\
\text { responsabilidad de la } \\
\text { educación del niño/ de la } \\
\text { niña? (B03) }\end{array}$ & No & 0,076 & Nula \\
\hline
\end{tabular}

Nota: los estilos que prevalecen en los perfiles determinados a partir del análisis de conglomerados de $\mathrm{k}$-medias, especificado en el inicio del apartado de Resultados.

De acuerdo con los cálculos, en el caso de la relación de B03, B11 y B31 no se demostró la existencia de la relación estadísticamente significativa con los diferentes Grupos (clústeres) marcados por dominancia de un estilo educativo. Sin embargo, hay que señalar la existencia de la relación entre estilos educativos y las variables B07, B09, B10, aunque el valor del coeficiente de contingencia nos señala que ésta es muy baja.

\section{Discusión y conclusiones}

Las familias muestran el valor social que le dan a la Educación de formas diversas. El estudio que se realizó en el marco del estudio de validación del instrumento EVALEF (Bisquert, 2017) incorporó variables que reflejan las diferentes dimensiones del VSE-Subjetivo, permitiendo explorar la hipótesis de que éste se vincula con el EEF de las familias. Los resultados del presente estudio muestran la asociación significativa en dos de las cuatro dimensiones que estructuran el concepto de VSE-Subjetivo definido por Jornet, et al (2011) y utilizado en este estudio, aunque con una intensidad baja (entre 0.103 y 0.155$)$.

Respecto a la Dimensión 1, relativa a expectativas y metas educativas, se encuentra asociación significativa entre los clusters establecidos por los EEF y la expectativa de estudios que padres y madres tienen para sus hijos, mostrando mayores expectativas el grupo con perfil que se decantó en la prueba prioritariamente por el Estilo Integral y complementariamente por el Democrático (9Int4Dem). De acuerdo con las investigaciones similares, el estilo educativo democrático permite mantener la relación afectiva y facilitar la autonomía al hijo y éste "pase a depender cada vez más de 
sí mismo por una acción más consciente y responsable" (Valdivia, 2007, p. 289). Las expectativas educativas pueden entenderse vinculadas a esa percepción de "control personal" de las propias acciones; el estilo Democrático, por tanto, se podría vincular con ese desarrollo de expectativas y metas educativas.

La Dimensión 2, relativa a la vinculación entre justicia social percibida y educación, se ha analizado a través de tres elementos. En dos de ellos (implicación en la escolarización de los hijos y satisfacción con sus resultados) se han identificado diferencias significativas, ambas de escasa intensidad, en el mismo sentido que el caso de la dimensión anterior. En el caso del tercer elemento (satisfacción con el tiempo dedicado a los hijos), con una valoración media inferior a la interior, las diferencias no fueron significativas. En todos los casos, el grupo No-Def es el que ofrece puntuaciones más bajas en las variables estudiadas, pudiéndose inferir que una menor claridad en la intervención educativa de los progenitores (grupo No-Def) se vincula con una menor atribución de valor social a la educación (aunque también podría haber intervenido en este grupo una mayor dificultad en la resolución de la prueba, que podría explicar la falta de respuesta a lo largo de la escala).

En resumen, a partir del análisis realizado para la Dimensión 2, podríamos hablar de una asociación entre algunos elementos de justicia social y educación, tales como implicación por parte de los padres en la escolarización de sus hijos y la satisfacción con los resultados escolares de éstos, siendo estos resultados coherentes con los de investigaciones previas. El estudio del Secretariado General Gitano (2001) citado en González Sala (2006) sobre los perfiles de las familias en situación de riesgo social afirma, en consonancia con nuestros resultados, que esas familias suelen mostrar una implicación pobre en relación con la escuela, señalando que "un $80 \%$ no acude a las reuniones del Consejo Escolar y un 70\% nunca ha solicitado una entrevista con el profesor de su hijo" (p. 20). Además, otros estudios sobre las familias de menores en dificultad social han mostrado una serie de factores que conducen a la exclusión social y situaciones de riesgo para el menor, entre esos factores se señalan analfabetismo de los padres, escasa implicación en la educación del menor, conflictividad familiar (Galante y Martín, 2000; González Sala, 2006). Por otro lado, Epstein (2002) propone que desde la administración educativa se organicen las actividades para implicar a los padres en el proceso educativo escolar con el fin de mejorar la eficacia educativa. Estas actividades pueden centrarse en intercambio de información entre la institución y el colectivo de padres y madres, para compartir los objetivos educativos, colaboración y voluntariado, toma de decisiones compartida.

Finalmente, en las otras dos dimensiones (valor diferencial de la educación, y obstáculos y facilitadores) no se han encontrado asociaciones significativas.

En conclusión, se trata de dos constructos, EEF y VSE-Subjetivo, complejos y difíciles de aprehender. La literatura muestra que el EEF Democrático se vincula con una mayor implicación en la educación de los hijos e hijos, y mejores resultados escolares. El EEF Integral, como profundización del Democrático, debería ir en el mismo sentido. Invertir, como familia, energía y tiempo en la educación de los hijos, apostar por explicitar los valores en la educación, y el valor del esfuerzo y la propia implicación en el éxito escolar, se vincula conceptualmente con la percepción subjetiva de que la educación tiene un valor social, un valor en el propio desarrollo personal, laboral y social. Los resultados de este estudio muestran esa asociación, pero sólo de un modo parcial, y de un modo menos intenso del esperado.

Por un lado, podemos afirmar que este estudio sirve de evidencia empírica de replicación de los estudios anteriores que pretenden relacionar sobre todo la implicación de los padres y madres en la educación de sus hijos. Por otro lado, creemos que es necesario profundizar en la búsqueda de otros elementos implicados, identificando diferentes indicadores para medir el VSE-Subjetivo que las familias atribuyen a la educación y depurando la medición y el análisis de los EEF. La reflexión de las familias sobre su forma de educar y los motivos que tienen para hacerlo (explícitos e implícitos; conscientes e inconscientes), y su reflexión sobre el valor que le dan a la educación, y el valor que transmiten a sus hijos e hijas, van a ser instrumentos fundamentales para mejorar esa implicación, y conseguir que la educación desarrolle al máximo su potencial como elemento de dinamización y desarrollo personal y social, especialmente si esta reflexión se plantea y dinamiza desde la colaboración familia-escuela. 


\section{Referencias bibliográficas}

Bisquert, M. (2017). Diseño y validación de un instrumento para evaluar los Estilos Educativos Familiares. EVALEF. Universidad de Valencia, Valencia.

Cánovas, P., Sahuquillo, P. M., Císcar, E. y Martínez, C. (2014). Estrategias de intervención socioeducativa con familias: Análisis de la orientación familiar en los servicios especializados de atención a la familia e infancia de la Comunidad Valenciana. Educación XXI, 17(2), 256288. Doi: 10.5944/educxx1.17.2.11491

Cánovas. P. y Sahuquillo, P. (2010). Educación y diversidad familiar: aproximación al caso de la monoparentalidad. Educatio Siglo XXI, 28(1), 109-126.

Cardona, M. L. (2011). Las Familias en los Estudios de Evaluación de Sistemas Educativos. Validación de un Cuestionario de Estilos Educativos Familiares. I Jornadas Doctorales de Castilla-La Mancha.

Epstein, J.L. (2002). School, Family, and Community Partnerships: Your Handbook for Action, $2^{\mathrm{a}}$ Ed. Washington, DC.: Corwin Press Inc.

González Sala, F. (2006). Estudio de los perfiles de las familias en situación de riesgo social : Programas de ayudas P.E.R y P.E.P. del Ayuntamiento de Valencia. Tesis doctoral, Universitat de València.

Jornet, J.M.; Perales Montolío, M.J. y Sánchez Delgado, P. (2011). El valor social de la educación: entre la subjetividad y la objetividad. Consideraciones para su evaluación. Revista Iberoamericana de Evaluación Educativa, 4(1), 51-77.

Lázaro Lorente, L. M. (2017). El impacto de la crisis económica global en la educación. Repercusiones sobre la educación superior, 2008-2014. Revista Latinoamericana de Educación Comparada, 8(11), 13-30.

Mourshed, M., Chijioke, C. y Barber, M. (2012). Cómo continúan mejorando los sistemas educativos de mayor progreso en el mundo. Chile: Preal, McKinsey \& Company.

OCDE (2018). Equity in Education: Breaking Down Barriers to Social Mobility. PISA, OECD Publishing, Paris. https://doi.org/10.1787/9789264073234-en

OCDE. (2015). Transforming our world: The 2030 agenda for sustainable development. París: OCDE.

Perales, M. J., Bisquert, M. y Sahuquillo, P. (2015, Septiembre). Definición del constructo de estilos educativos familiares para el cuestionario EVALEF (Evaluación de Estilos Educativos Familiares). Comunicación presentada en el IV Congreso Internacional de Atención Temprana y Educación familiar (CLATEF). Santiago de Compostela.

Sáncho-Álvarez, C., Jornet, J.M. y González-Such, J. (2017). Una aproximación al valor social subjetivo de la educación en Iberoamérica. Evidencias de validez entre Argentina, España y República Dominicana. Perfiles Educativos, 39(157), 14-33.

Schraad-Tischler, D. y Schiller, C. (2016). Social Justice in the EU - Index Report 2016. Social Inclusion Monitor Europe. Gütersloh (Alemania): Bertelsmann Stiftung.

Secretariado General Gitano (2001). Gitanos: pensamiento y cultura. Dossier Educación, 11, octubre. Madrid, SGG.

Valdivia, M.C. (2007). Los estilos educativos en la educación Familiar. Revista Comillas, 65(126), 267291. 
Para citar este artículo

Perales Montolío, M.J., Bisquert Martínez, M. \& Bakieva, M. (2018). Relación entre estilos educativos familiares y el valor social subjetivo de educación: reflexiones a partir de un estudio. Revista Fuentes, 20(2), 51-73. [Fecha de consulta: dd/mm/aa]. doi: http://dx.doi.org/10.12795/revistafuentes.2018.v20.i2.04 